\title{
Commonly Occurring Bacteria in Diabetic Foot Infections and their Sensitivity to various Antibiotics
}

\author{
Muneeb-ullah ${ }^{1}$, Awais Saeed Abbasi ${ }^{2}$, Seemab Niaz ${ }^{3}$, Wajiha Mahjbeen 4 \\ 1,2 Registrar, Accident and Emergency Department, Dr. Akbar Niazi Teaching Hospital (DANTH), Islamabad. \\ ${ }^{3}$ Post Graduate Trainee, PIMS Hospital, Islamabad. \\ ${ }^{4}$ Associate Professor, Chemical Pathology, DANTH, Islamabad
}

\section{ABSTRACT}

Background: Diabetic foot infections are a common cause of morbidity in type $1 \& 2$ Diabetes mellitus. The selection of appropriate empirical treatment is thus essential while treating such patients. The main objective of this study was to find out most prevalent bacteria and their sensitivity to various antibiotics in patients with diabetic foot infections.

Material and Methods: This cross-sectional study was conducted in the Department of Surgery, Pakistan Institute of Medical Sciences (PIMS), Islamabad from January 2017 to December 2017. A total 118 patients were included through consecutive sampling technique. Samples were collected in the form of swab, pus or tissue material and were cultured on blood agar and microorganisms were identified using standard microbiological methods. Antimicrobial sensitivity was also checked. Statistical Package for Social Sciences (SPSS) version 22 was used to analyze data.

Results: Out of 118 patients, $72 \%(n=85)$ were males while $28 \%(n=33)$ were females. Cultures of 105 patients were positive and most common organisms isolated were S. aureus (32.4\%; $n=34)$, E. coli (17.1\%; $n=18)$, P. aeruginosa (14.3\%; $n=15)$, Polymicrobials $(14.3 \% ; n=15)$ and $K$. pneumoniae $(7.6 \% ; n=8)$. Antibiotics to which organisms were most sensitive included Piperacillin/Tazobactam (69.5\%; n=73), Imipenem (55.2\%; n=58), Amikacin (43.8\%; n=46), Vancomycin (40\%; n=42) and Levofloxacin (38.1\%; n=40).

Conclusion: Most common organisms causing diabetic foot infections in our study were S. aureus, E. coli, P. aeruginosa, Polymicrobial and Klebsiella. Overall most sensitive antibiotics to these organisms included Piperacillin/Tazobactam, Imipenem, Amikacin, Vancomycin and Levofloxacin.

Key words: Antibiotics, Diabetic foot infections, Escherichia coli, Pseudomonas aeruginosa, Piperacillin/Tazobactam, Staphylococcus aureus

$\begin{array}{lll}\text { Authors' Contribution: } & \text { Correspondence: } & \text { Article info: } \\ 1,2 \text { Conception, synthesis, planning of } & \text { Muneebullah } & \text { Received: March 24, 2018 } \\ \text { research and manuscript writing }{ }^{3} & \text { Email: muneebullah@gmail.com } & \text { Accepted: January 10, 2019 }\end{array}$
Interpretation, discussion, Active participation in data collection

${ }^{4}$ Data analysis.

Cite this article. Muneeb-ullah, Abbasi AS, Niaz S, Mahjbeen W. Commonly occurring bacteria in diabetic foot infections and their sensitivity to various antibiotics. J Islamabad Med Dental Coll.2019; 8(1):8-12

Funding Source: Nil Conflict of Interest: Nil

\section{Introduction}

The average worldwide prevalence of type 2 Diabetes Mellitus in adults is $6.4 \%$. It ranges from 3.8 to $10.2 \%$ among different regions of Pakistan. Rates of undiagnosed diabetes may be as high as $50 \%$ in some areas. ${ }^{1}$ Incidence of Type 1 Diabetes Mellitus also vary worldwide with highest reported incidence in Finland and Sardinia (37- 65 per 100,000) and lowest in China and
Venezuela (0.1-1.9 per 100,000).2,3 Foot related problems are an important cause of morbidity in patients with diabetes mellitus. Lifetime risk of foot ulcers for diabetic patients (type 1 or 2) may be as high as $25 \% .{ }^{4}$ Neuropathy, peripheral vascular disease (PVD) and poor glycemic control are among the important risk factors responsible for development of diabetic foot infection. ${ }^{5}$ 
Sensory neuropathy causes diminished perception of pain and temperature that leads to poor recognition of injury to the feet. Autonomic neuropathy causes reduced sweat secretion resulting in dry, cracked skin that facilitates the entry of microorganisms. Motor neuropathy leads to foot deformities leading to pressure induced soft tissue damage. Most of the diabetic foot infections are polymicrobial and are variable depending on the extent of involvement. $^{5}$

Host defense and neutrophil functions are impaired by hyperglycemia. Trauma in patients with one or more of these risk factors precipitate development of wounds that are slow to heal and predispose to secondary infection. Three key steps involved in evaluation of a patient with diabetic foot infection are: 1) identification of risk factors, 2) determination of extent and severity of infection and 3 ) assessment of microbial etiology. ${ }^{5}$ For preventive and monitoring strategies, certain risk categorization systems can be used. One such system is developed by International Working Group on the Diabetic Foot that stratifies patients as follows: ${ }^{6}$ ) No evidence of neuropathy, 1) neuropathy present but no evidence of foot deformity or PVD, 2) neuropathy with evidence of deformity or PVD, 3) history of foot ulceration or lower extremity amputation. At least 2 of the following i.e. erythema, warmth, tenderness or swelling should be present to make a presumptive diagnosis of diabetic foot infection. Osteomyelitis is likely to be present if bone can be seen at the floor of deep ulcer. ${ }^{7}$ Aerobic gram-positive cocci which include Staphylococcus aureus (S. aureus), Streptococcus agalactiae (S. agalactiae), Streptococcus pyogenes (S. pyogenes) and coagulase negative staphylococci are mostly responsible for superficial diabetic foot infections (cellulitis and infected ulcers in antibiotic-naive patients). ${ }^{8}$

Deep and chronically infected ulcers and those that are previously treated with antibiotics, are more likely to be polymicrobial and in addition to above mentioned microorganisms; involve Enterococci, Enterobacteriaceae, Pseudomonas aeruginosa ( $P$. aeruginosa), and anaerobes. ${ }^{8}$ Wounds with extensive local inflammation, necrosis, malodorous drainage, necrosis, or gangrene with signs of systemic toxicity should be presumed to have anaerobic organisms in addition to the above pathogens. ${ }^{8}$ Microbiological spectrum also differs by geographic location; with gram-negative pathogens predominating in the sub-tropical climates of Africa and Asia, in contrast to gram-positive organisms in the western parts of the world. ${ }^{9}$ Current study is aimed at identifying the common pathogens involved in diabetic foot infections in our set-up and their susceptibility to commonly used antimicrobial therapy that may guide in selection of effective empiric treatment.

\section{Material and Methods}

This was a cross sectional study, conducted in the Department of Surgery, Pakistan Institute of Medical Sciences (PIMS), Islamabad. Duration of study was one year, from January 2017 to December 2017. Patients, presenting in the outpatient department, emergency or ward, qualifying the criteria of PEDIS (Perfusion, Extent, Depth, Infection and Sensation) system of diabetic foot classification were included in the study. ${ }^{10}$ This study was approved by the ethics committee of the hospital and written informed consent was obtained from all the patients prior to enrolment in this study. A total of 118 patients presenting with signs and symptoms suggestive of Diabetic Foot Infection were included in this study through consecutive sampling technique.

Sample size was calculated through WHO sample size calculator by using $95 \%$ confidence interval, $80 \%$ power of study and $7.4 \%$ prevalence of diabetic foot ulcer in Pakistan. ${ }^{11}$ Calculated sample size was 106 diabetic patients. In order to overcome the possibility of dropouts, total 118 patients were included in the study. Collected samples included swabs, pus and tissue material after cleansing with non-antimicrobial substance. At the time of sample collection, no patient was on antimicrobial therapy. Samples were sent promptly to microbiology laboratory where they were cultured on blood agar and MacConkey agar plates. Organisms were further identified using respective biochemical tests according to standard microbiological protocols. Bacteria that were cultured included S. aureus, E. coli, P. aeruginosa, Proteus, Klebsiella pneumoniae, methicillin resistant $\mathrm{S}$. aureus, Staphylococcus epidermidis, Acinetobacter, S. viridans and Enterobacter. A Polymicrobial group was added when culture was positive for two or more 
organisms. Isolates were tested for susceptibility to commonly used antimicrobial therapy. Antibiotics that were included in culture sensitivity included Piperacillin/Tazobactam, Linezolid, Ceftriaxone, Vancomycin, Imipenem, Meropenem, Levofloxacin, CoAmoxiclav, Cefoperazone ISulbactam, Amikacin, Clindamycin, Ceftazidime, Ciprofloxacin, Tigecycline, Chloremphenicol, Tobramycin and Cefoxitin. Statistical Package for Social Sciences (SPSS) version 22 was used to analyze data. The categorical data is presented in frequencies and percentages i.e. gender frequency, most common organisms and most sensitive antibiotics.

\section{Results}

A total of 118 patients were included in this study with a mean age of $53 \pm 9.8$ years. Out of 118 patients, 85 were males $(72 \%)$, and 33 were females (28\%). About 105 patients $(89 \%)$ tested positive for bacterial growth, while 13 patients (11\%) had no organism growth on culture. Of the culture positive patients, $71.4 \%$ were males and $28.6 \%$ were females. Cultured organisms were divided into 11 groups. First five organisms isolated in 105 culture positive patients in descending order were, $\mathrm{S}$. aureus $(32.4 \% ; n=34)$, E. coli $(17.1 \% ; n=18)$, P. aeruginosa (14.3\%; $n=15)$, Polymicrobial (14.3\%; $n=15)$ and $K$. pneumoniae $(7.6 \% ; n=8)$ (Table I). Top five antibiotics to which organisms were sensitive in descending order were Piperacillin/Tazobactam (69.5\%), Imipenem (55.2\%), Amikacin (43.8\%), Vancomycin (40\%) and Levofloxacin (38.1\%) (Table II).

\begin{tabular}{|c|c|c|c|}
\hline \multicolumn{4}{|c|}{$\begin{array}{l}\text { Table I: Frequency and Percentage of organisms isolated in } \\
\text { culture positive patients }(n=105)\end{array}$} \\
\hline $\begin{array}{l}\text { Sr. } \\
\text { No }\end{array}$ & Organism & Frequency & Percentage \\
\hline 1 & Staphylococcus aureus & 34 & $32.4 \%$ \\
\hline 2 & Escherchia coli & 18 & $17.1 \%$ \\
\hline 3 & Pseudomonas aeruginosa & 15 & $14.3 \%$ \\
\hline 4 & Polymicrobial & 15 & $14.3 \%$ \\
\hline 5 & Klebsiella pneumoniae & 8 & $7.6 \%$ \\
\hline 6 & Proteus & 5 & $4.8 \%$ \\
\hline 7 & $\begin{array}{l}\text { Methicillin resistant } \\
\text { staphylococcus aureus }\end{array}$ & 3 & $2.9 \%$ \\
\hline 8 & Staphylococcus epidermidis & 3 & $2.9 \%$ \\
\hline 9 & Acinitobacter & 2 & $1.9 \%$ \\
\hline 10 & Streptococcus viridans & 1 & $1 \%$ \\
\hline 11 & Enterobacter & 1 & $1 \%$ \\
\hline
\end{tabular}

On an individual basis, S. aureus $(n=34)$ was most sensitive to vancomycin $(61.8 \% ; n=21)$, E. coli $(n=18)$ was most sensitive to Piperacillin/Tazobactam $(88.9 \% ; n=16)$, $P$. aeruginosa $(n=15)$ was most sensitive to Piperacillin/Tazobactam (100\%; $n=15)$, Polymicrobial $(n=15)$ was most sensitive to Piperacillin/Tazobactam and Amikacin (60\%; $n=9)$ and K. pneumoniae $(n=8)$ was most sensitive to Piperacillin/Tazobactam $(75 \% ; n=6)$ (Table III).

\begin{tabular}{|c|l|c|c|}
\hline \multicolumn{5}{|c|}{$\begin{array}{c}\text { Table II: Frequency and Percentage of antibiotic sensitivity } \\
\text { against organisms isolated (n=105) }\end{array}$} \\
\hline Sr No & \multicolumn{1}{|c|}{ Antibiotic } & Frequency & Percentage \\
\hline 1 & Piperacillin/Tazobactam & 73 & $69.5 \%$ \\
\hline 2 & Imipenem & 58 & $55.2 \%$ \\
\hline 3 & Amikacin & 46 & $43.8 \%$ \\
\hline 4 & Vancomycin & 42 & $40 \%$ \\
\hline 5 & Levoflaxacin & 40 & $38.1 \%$ \\
\hline 6 & Cefoperazone/Sulbactam & 34 & $32.4 \%$ \\
\hline 7 & Co-amoxiclav & 33 & $31.4 \%$ \\
\hline 8 & Ceftriaxone & 22 & $21 \%$ \\
\hline 9 & Linezolid & 16 & $15.3 \%$ \\
\hline 10 & Meropenem & 16 & $15.3 \%$ \\
\hline 11 & Tobramycin & 13 & $12.4 \%$ \\
\hline 12 & Ciprofloxacin & 6 & $5.7 \%$ \\
\hline 13 & Tigecycline & 5 & $4.8 \%$ \\
\hline 14 & Clindamycin & 4 & $3.8 \%$ \\
\hline 15 & Chloremphenicol & 4 & $3.8 \%$ \\
\hline 16 & Ceftazidime & 1 & $1.8 \%$ \\
\hline 17 & Cefoxitin & & $1 \%$ \\
\hline
\end{tabular}

\section{Discussion}

Our study shows that there is a male predominance in patients of diabetic foot with male to female ratio of 2.5:1. Other studies carried out in Pakistan either show higher male predominance of 4:1 for diabetic patients at Nishtar Hospital, Multan ${ }^{12}$ and CMH Peshawar, ${ }^{13}$ or comparable ratio of 2.3:1.14 Male predominance can be due to males working outdoors exposed to contaminated surroundings compared to females performing household chores. Moreover, females in general are more concerned about their health and adopt preventive strategies. Our study shows mean age of $53 \pm 9.8$ years, which is in agreement to a study done on 73 patients in Karachi with a mean age of $52.7 \pm 9.4$ years. ${ }^{15}$ According to another study carried out in Nishtar Hospital Multan, the most commonly affected age group was also 50 to 60 years. ${ }^{12}$ The logical explanation for affecting older individuals is that Diabetes is usually diagnosed at a later age with multiple 


\begin{tabular}{|c|l|c|c|c|c|c|}
\hline \multicolumn{7}{|c|}{ Table III: Top five common organisms and their sensitivity to different antibiotics } \\
\hline $\begin{array}{c}\text { Sr } \\
\text { No }\end{array}$ & Antibiotic Sensitivity & $\begin{array}{c}\text { S. Aureus } \\
(\mathrm{n}=\mathbf{3 4 )}\end{array}$ & $\begin{array}{c}\text { E. Coli } \\
(\mathrm{n}=18)\end{array}$ & $\begin{array}{c}\text { Pseudomonas } \\
(\mathrm{n}=\mathbf{1 5})\end{array}$ & $\begin{array}{c}\text { Polymicrobial } \\
(\mathrm{n}=\mathbf{1 5})\end{array}$ & $\begin{array}{c}\text { Klebsiella } \\
(\mathrm{n}=\mathbf{8})\end{array}$ \\
\hline 1 & Piperacillin/ Tazobactam & $55.9 \%(\mathrm{n}=19)$ & $88.9 \%(\mathrm{n}=16)$ & $100 \%(\mathrm{n}=15)$ & $60 \%(\mathrm{n}=9)$ & $75 \%(\mathrm{n}=6)$ \\
\hline 2 & Imipenem & $52.9 \%(\mathrm{n}=18)$ & $66.7 \%(\mathrm{n}=12)$ & $80 \%(\mathrm{n}=12)$ & $40 \%(\mathrm{n}=6)$ & $37.5 \%(\mathrm{n}=3)$ \\
\hline 3 & Amikacin & $26.5 \%(\mathrm{n}=9)$ & $44.4 \%(\mathrm{n}=8)$ & $40 \%(\mathrm{n}=6)$ & $60 \%(\mathrm{n}=9)$ & $75 \%(\mathrm{n}=6)$ \\
\hline 4 & Vancomycin & $61.8 \%(\mathrm{n}=21)$ & $22.2 \%(\mathrm{n}=4)$ & $33.3 \%(\mathrm{n}=5)$ & $26.7 \%(\mathrm{n}=4)$ & $12.5 \%(\mathrm{n}=1)$ \\
\hline 5 & Levoflaxacin & $50 \%(\mathrm{n}=17)$ & $27.8 \%(\mathrm{n}=5)$ & $46.7 \%(\mathrm{n}=7)$ & $6.7 \%(\mathrm{n}=1)$ & $12.5 \%(\mathrm{n}=1)$ \\
\hline
\end{tabular}

co-morbidities, poor diabetic control and nutritional deficiencies. The most common groups of organisms identified in this study are S. aureus, E. coli, P. Aeruginosa and Polymicrobial. This was also seen in a study done in Khyber Pakhtunkhwa where S. aureus and E. coli were the most common organisms in diabetic foot patients. ${ }^{16}$ In another study Staphylococcus aureus was the most prevalent organism constituting $23.16 \%$ of the organisms isolated followed by Escherichia coli (17.89\%) and Klebsiella (12.63\%). ${ }^{15}$ Proteus, S. aureus, Klebsiella and $P$. aeruginosa were the most widely recognized microorganisms of diabetic foot infections. ${ }^{17}$ Another study showed $S$. aureus being the most commonly isolated organism. ${ }^{18,19}$ Our findings are comparable with other studies conducted in this region. In our study, organisms are most sensitive to Piperacillin/Tazobactam, Imipenem, Amikacin, Vancomycin and Levofloxacin. In a study on diabetic foot ulcers in Jinnah Postgraduate Medical Center, Karachi organisms were most sensitive to Meropenem, effective in $95 \%$ patients. ${ }^{15}$

The specific antibiotic given against culture sensitive organisms helps in prevention of drug resistance, more accurate management and speedy recovery rather than empirical therapy. So, each organism being more sensitive to specific antibiotic should be treated with antibiotic of choice accordingly. Culture sensitivity should be done regularly to identify the organism and start proper antibiotic regimen. Hospital based studies can also help in maintaining an antibiogram, which should be periodically updated for devising antibiotic protocols for effective treatment of bacterial infections.

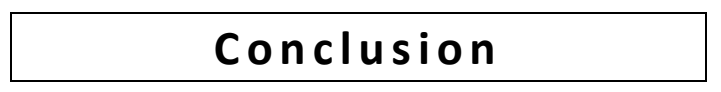

Diabetic foot infections are common in older age group with male predominance. Most common infecting organisms included S. aureus, E. coli, P. aeruginosa, Polymicrobial and Klebsiella with Piperacillin/ Tazobactam, Imepenem, Amikacin, Vancomycin and Levofloxacin emerging as the most sensitive antibiotics in our diabetic patients.

\section{References}

1. Ali A, Abbasi AS, Mushtaq $S$, Azim $S$, Jamil M. A Comparative Study of Waist Circumference, WaistHip Ratio and BMI in Diabetics and Non-Diabetics. Ann. Pak. Inst. Med. Sci. 2017:13(1):27-34.

2. Harjutsalo V, Sund R, Knip M, Groop PH. Incidence of type 1 diabetes in Finland. JAMA 2013; 310(4):427-428.

3. Weng J, Zhou Z, Guo L, Zhu D, Ji L, Luo X, et al. Incidence of type 1 diabetes in China, 2010-13: population-based study. BMJ 2018; 360: j5295.

4. Boulton A, Armstrong D, Albert S, Frykberg R, Hellman R, Kirkman M, et al. Comprehensive foot examination and risk assessment. Endocrine Practice. 2008 ;14(5):576-83.

5. Lipsky BA, Berendt AR, Deery HG, Embil JM, Joseph WS, Karchmer AW, et al. Diagnosis and treatment of diabetic foot infections. Clin Infect Dis 2004; 39(7):885.

6. Bus SA, van Netten JJ, Lavery LA, Monteiro-Soares $M$, Rasmussen $A$, Jubiz $Y$, et al. IWGDF guidance on the prevention of foot ulcers in at-risk patients with diabetes. Diabetes Metab Res Rev 2016; 32(1):16.

7. Grayson ML, Gibbons GW, Balogh K, Levin E, Karchmer AW. Probing to bone in infected pedal ulcers. A clinical sign of underlying osteomyelitis in diabetic patients. JAMA 1995; 273(9):721-3.

8. Zubair M, Malik A, Ahmad J. Clinico-microbiological study and antimicrobial drug resistance profile of 
diabetic foot infections in North India. Foot (Edinb) 2011; 21(1):6-14.

9. Hatipoglu M, Mutluoglu M, Uzun G, Karabacak E, Turhan V, Lipsky BA. The microbiologic profile of diabetic foot infections in Turkey: a 20-year systematic review: diabetic foot infections in Turkey. Eur J ClinMicrobiol Infect Dis 2014; 33(6):871-8.

10. Schaper NC. Diabetic foot ulcer classification system for research purposes: a progress report on criteria for including patients in research studies. Diabetes Metab Res Rev 2004; 20(1): S90.

11. Zhang $P$, Lu J, Jing Y, Tang S, Zhu D, Bi Y. Global epidemiology of diabetic foot ulceration: a systematic review and meta-analysis. Ann med. 2017 Feb 17;49(2):106-16.

12. Khan $A B G M$, Bhatti $A$, Qureshi KH. Diabetic foot; surgical management. Professional Med J.2012;19(1):06-10.

13. Mishwani $A H$, Kiyani KA. Surgical management of diabetic foot and role of UT (university of Texas) classification.Pak Armed Forces Med J. 2011;61(3):367-71.

14. Imran M, Mahmood Z, Nadeem M, Tahir Ch, Tashah. Pattern of diabetic foot lesions and surgical procedures for management. Pak $\mathrm{J}$ Med Health Sci.2011;5(1):81-4.
15. Nageen A. The Most Prevalent Organism in Diabetic Foot Ulcers and Its Drug Sensitivity and Resistance to Different Standard Antibiotics.J Coll Physicians Surg Pak. 2016;26(4):293-6.

16. Ayub R, Raza SS, Shafiullah, Ahsan J, Hussain AK, Nadeem MD. Bacterial Culture Isolates from Infected Diabetic Foot Tissue Specimens and Their Sensitivity To Antimicrobial Agents. J Med Sci. 2016;24(4):2734.

17. Rahimoon AG, Alam MT, Talpur MS. DIABETIC FOOT INFECTION; Frequency of microbes and antimicrobial sensitivity pattern attertiary care hospital, Karachi. Professional Med J. 2015;22(11):1415-22.

18. Lipsky BA, Berendt AR, Cornia PB, Pile JC, Peters EJ, Armstrong DG, et al. Infectious Diseases Society of America clinical practice guideline for the diagnosis and treatment of diabetic foot infections. Clin Infect Dis; 2012: e132-e173.

19. Roberts $A D$, Simon GL. Diabetic foot infections: the role of microbiology and antibiotic treatment. SeminVasc Surg. 2012; 25(2):75-81. 